Editorial

\title{
Sublingual route during perioperative and chronic pain management
}

Volume 3 Issue I - 2015

\section{Editorial}

The route of administration of drugs is a concern in perioperative period and for chronic pain management. The route may be invasive or noninvasive depending on the drug, its preparation and indication for its use. The patient acceptability is better with noninvasive routes specially oral routes. However in certain cases, this oral route may not be feasible due to patient clinical condition and mandates alternative route. ${ }^{1-4}$ Usually in these situation invasive route like intravascular administration remains the main stay. Here sublingual route may be considered an alternate option for drug administration. Sublingual route for drug therapy has found its role in cardiovascular drugs, steroids, barbiturates and enzymes. ${ }^{5-8}$

Systemic drug delivery through the sublingual route had emerged from the desire to provide immediate onset of pharmacological effect. ${ }^{9-11}$ This route offers a number of advantages such as rapid onset, ease of administration, avoidance of first pass metabolism so it provides better bioavailability. It is a non invasive and painless route of administration. It is also suitable for patients with nausea, vomiting, dysphagia, gastrointestinal disturbance such as obstruction. The systemic drug delivery after sublingual route is fast as drug directly reaches in to the blood stream through the reticulated vein on ventral surface of tongue and floor of mouth via facial veins, internal jugular vein, and brachiocephalic vein. ${ }^{3}$ It occurs through passive diffusion into the lipoidal membrane of the oral cavity. The sublingually administered drugs are absorbed 3 to 10 times greater as compared to conventional oral route. This is because of high permeability and the rich blood supply of the sublingual region. ${ }^{3}$ The drug delivered through the sublingual route needs to have specific properties and is affected by physicochemical properties of the drug and the patient oral condition. The drug should be lipophillic, unionized, soluble in aqueous buccal fluids, binds poorly to mucosa and low molecular weight. ${ }^{1}$ The acidic environment due to salivary glands and accompanying vasodilation may also facilitates absorption via sublingual route. ${ }^{3}$ The various advantage of sublingual routes includes rapid onset of action. It avoids first pass hepatic metabolism and thus higher bioavailability. The drugs through sublingual route avoids gastric degradation due to $\mathrm{pH}$ changes. However sublingual route may not be used for prolonged administration. Sustained drug delivery may not be achieved via this route. It may interfere with eating and drinking during as drug needs to be retained for some time when given via sublingual route. It may also be associated with unpalatability, burning sensation and ulceration. Smoking causes oral vasoconstriction and thus affects the drug absorption. ${ }^{4}$ The medical preparation for sublingual administration includes:

a. Sublingual tablets: Easily melts in mouth, dissolve rapidly and with little or no residue e.g. Nitroglycerine, ondansetron, buprenorphine

b. Multipurpose tablets: Soluble tablets for either oral/sublingual administration e.g. Hydromorphone.

\author{
Rakesh Garg,' Shalini Saini² \\ 'Department of Onco-Anesthesiology and Palliative Medicine, \\ Assistant Professor, India \\ ${ }^{2}$ Department of Onco-Anesthesiology and Palliative Medicine, \\ Senior Resident, India
}

Correspondence: Rakesh Garg, Department of Onco-

Anesthesiology and Palliative Medicine, DR BRAIRCH All India Institute of Medical Sciences, Ansari Nagar, New Delhi - I 10029 , India, Tel +91-9868398335, +91-9810394950,

Email drrgarg@hotmail.com

Received: September 26, 2015 | Published: October 06, 2015

c. Sublingual drops: Concentrated solution to be dropped under tongue.

d. Lozenges: Combination of sublingual, buccal, and oral administration e.g. fentanyl lozenges.

Patients requires drugs in perioperative care for sedation, anxiolysis ease and pain management, in addition to other therapeutic management. ${ }^{6}$ Premedication is paramount for better patient comfort. The invasive route may further lead to anxiety and discomfort. Oral and sublingual route may be considered most acceptable to the patients. Also, sublingual route may be beneficial in children wherein they may not cooperate for invasive vascular access. ${ }^{6}$ At times inability to take orally due to medical reasons may warrant alternative routes for drug administration. Inability to accept orally may also be seen in elderly, children, and patients who are mentally retarded, uncooperative, nausea / vomiting or pain on swallowing like mucositis. ${ }^{3}$

Opioids can be administered by as many routes as no other class of drugs. Sublingual route have argued for its superiority as defined by rapidity of onset, intensity and duration of analgesia, and lesser magnitude of opioid-related side effects. ${ }^{8-11}$ The breakthrough pain management for chronic pain management may be better managed with sublingual route as oral ingestion may have delayed onset and thus poor patient satisfaction. ${ }^{11}$ Buprenorphine, a potent, synthetic, mixed agonist-antagonist with long half life, is readily absorbed by sublingual rote. It has high first pass metabolism. However, systemic bioavailability is $50 \%$ after sublingual administration. It provides good preoperative sedation, intraoperative as well as postoperative analgesia. It also decreases the requirement of other analgesics. ${ }^{11}$ In palliative medicine, pain management of homebound dying hospice patients who are no longer able to swallow, sublingual route plays a vital role. ${ }^{11}$ Morphine, most widely used opioid has also received the most research attention. Pharmacokinetic data, however, were equivocal. Some concluded that poor absorption made morphine unsuitable for sublingual administration ${ }^{8}$ the preponderance of available data does not support assertions of clinical superiority of the sublingual route over the oral or other routes of administration for the most commonly used opioids for breakthrough pain: morphine, 
oxycodone, and hydromorphone. ${ }^{8}$ Morphine, oxycodone, and hydromorphone are strongly hydrophilic drugs. Lipophilic drugs tend to be absorbed best through the sublingual mucosa. ${ }^{8}$ In these regards, methadone, sufentanil, and buprenorphine hold promise to be effective due to high degrees of lipophilicity and potency, and availability in concentrated forms. Thinking about role of sublingual morphine in palliative care raises few questions. Will sublingual opiods help in baseline or episodic pain? Morphine and oxycodone have been the traditional opioids for this purpose, but data support that rapidity of onset is not robust and these agents have potency limitations. Methadone and buprenorphine might function well in this capacity due to rapid absorption. But, data is required to assess the role of sublingual buprenorphine in episodic cancer pain, taking into account it's ceiling effect.

Ketamine is a short acting anaesthetic that has analgesic properties at subanaesthetic doses. In palliative care settings, it is primarily used for neuropathic pain which is unresponsive or poorly responsive to first-line analgesics (which may include one or more of opioid drug, NSAID, tricyclic antidepressant, or anticonvulsant). ${ }^{11}$ It has also been used for phantom limb. It acts through inhibition of N-methylD-aspartate receptors. Ketamine also interacts with cholinergic and opiate receptors and possibly inhibits the synaptic re-uptake of monoamines. Powerful synergism arises from the combination of morphine with low doses of ketamine probably due to some opiod activity of ketamine. Ketamine vials which are licensed for injection can also be administered sublingually. Oral solution of ketamine in dose of $2.5 \mathrm{mg}-5 \mathrm{mg}$ of ketamine is given sublingually. Sublingual ketamine has a rapid onset peak blood level therefore it may be useful for rapid analgesic effect. However there is a potential for dysphoric type side effects.

\section{Conclusion}

To conclude, sublingual route may be considered as alternate route with rapid and more efficient drug release with quick onset of action. It may be more convenient to special age groups like children and elderly. It needs further exploration and more research for sublingual drug formulations for drugs in perioperative management. Despite many uses, sublingual route remains unexplored, yet potential route for an anaesthesiologist in perioperative and pain care settings.

\section{Conflicts of Interests}

The authors do not have any Conflict of interests.

\section{Acknowledgments}

None.

\section{Funding}

None.

\section{References}

1. Bind AK, Gnanarajan G, Kothiyal P, et al. A review: sublingual route for systemic drug delivery. Int J Drug Res Tech 2013;3(2):310-336.

2. Nibha KP, Pancholi SS. An Overview on: sublingual route for systemic drug delivery. Int J Res Pharama Biomed Sci 2012;3(2):913-923.

3. Narang N, Sharma J. Sublingual mucosa as a route for systemic drug delivery. Int J Pharm Pharm Sci. 2011;3(2):18-22.

4. Sung YF. Risks and benefits of drugs used in the management of postoperative nausea and vomiting. Drug Safety. 1996;14(3):181-197.

5. Baines D. Postoperative nausea and vomiting in children. Paediatr Anaesth. 1996;6:7-14.

6. Gupta S, Gadani H, Kedia S. Is premedication with midazolam more effective by the sublingual than the oral route? Anesth Essays Res. 2011;5(1):43-47.

7. Weinberg DS, Inturrisi CE, Reidenberg B, et al. Sublingual absorption of selected opioid analgesics. Clin Pharmacol Ther. 1988;44(3):335-343.

8. Tassinari D, Masi A, Sartori S, et al. Atypical absorption of morphine sulfate through oral mucosa: An unusual case of acute opioid poisoning. J Pain Symptom Manage. 1995;10(5):405-407.

9. Wadibia EC. Antiemetics. South Med J. 1999;92(2):162-165.

10. Wagner DS, Gauger V, Chiravuri D, et al. Ondansetron oral disintegrating tablets for the prevention of postoperative vomiting in children undergoing strabismus surgery. Ther Clin Risk Manag. 2007;3(4):691-694.

11. Reisfiled GM, Wilson GR. Rational use of sublingual opioids in palliative medicine. Journal Pall Med. 2007;10(2):465-475. 